\title{
Untersuchungen über das Zuckerspektrum in Honigblaseninhalt und Honig
}

\author{
H Pechhacker 1, W Praznik 2, J Klaus 2
}

\author{
${ }^{1}$ Höhere Bundeslehr- und Versuchsanstalt für Wein- und Obstbau mit \\ Institut für Bienenkunde, Abteilung Bienenzüchtung, A-3293 Lunz am See \\ ${ }^{2}$ Institut für Chemie der Universität für Bodenkultur, Wien, Österreich
}

(Eingegangen 2 Mai 1990; angenommen 18 Juli 1990)

\begin{abstract}
Zusammenfassung - Durch die Bestimmung der botanischen Herkunft, der Zuckermenge und des Zuckerspektrums des Honigblaseninhaltes konnte zwischen den meisten bzw wichtigsten Trachtpflanzen ein Unterschied auch im Zuckerspektrum festgestellt werden. Besonders deutlich war der Unterschied zwischen Honigtau "flüssig" und Honigtau "kristallisiert". Honigtau "flüssig" enthält 49,42 $\pm 5,56 \%$ Saccharose und 19,99 $\pm 4,10 \%$ Melezitose, bei Honigtau "kristallisiert" ist das Verhältnis umgekehrt. Unter Berücksichtigung der Invertierung kann auch aus dem Zuckerspektrum des Honigs ein gewisser Rückschluß auf die botanische Herkunft dieses Honigs gezogen werden.
\end{abstract}

Zuckerspektrum / Honig / Honigblaseninhalt / botanische Herkunft

\section{EINLEITUNG}

Es ist anzunehmen, daß der Honigblaseninhalt in seiner Zusammensetzung weitgehend mit dem Nektar oder Honigtau von der bestimmten Pflanze bzw Honigtauerzeugern übereinstimmt, von welchen die Bienen den Rohstoff für den Honig gesammelt haben, da die Zeit zwischen dem Sammeln und der Probennahme kurz ist.

Durch den Einfluß von Enzymen kann sich das Zuckerspektrum aber sehr schnell ändern. Dadurch könnten bereits während des Eintrages enzymatische Veränderungen des Zuckerspektrums stattfinden. Es liegen bisher allerdings weder Untersuchungen des Zuckerspektrums des Honigblaseninhaltes, noch ein Vergleich der Zuckerspektren des Nektars (Honigtaues) und des Honigblaseninhaltes gleicher botanischer Herkunft vor.
Die Zuckerspektren des Nektars der untersuchten Pflanzen sind verschieden (Sihag und Kapil, 1984; Low et al, 1988; Handel et al, 1972) und selbst in den blütenreinen Honigen noch deutlich ausgeprägt (Maurizio, 1964).

Auch der Honigtau unterscheidet sich zwischen den einzelnen Honigtauerzeugern bzw Wirtspflanzen (Gölz, 1981; Liebig, 1979; Maurizio, 1985 in Kloft et a).

In der vorliegenden Arbeit wurden folgende Fragen untersucht : Kann durch die Unterschiede im Zuckerspektrum des Honigblaseninhaltes die Trachtherkunft erkannt werden ? Besteht ein Zusammenhang zwischen dem Zuckerspektrum des Honigblaseninhaltes und dem Zuckerspektrum des Honigs desselben Volkes unter bestimmten Versıchsbedingungen ? 


\section{METHODE}

\section{Versuchsvolk}

Im Rahmen eines Versuchsprogrammes wurde von einem Bienenvolk zu Beginn der Waldtracht am 30 Juni 1987 in Lunz ein Kunstschwarm gebildet und ohne jede Fütterung auf Mittelwände gesetzt. Diesem Volk wurde jeder Vorrat an Pollen und Honig und auch jede Brut genommen. Dadurch waren von einer vorhergehenden Tracht keine Pollen- und Honigvorräte mehr vorhanden.

\section{Probennahme}

Von diesem so erstellten Kunstschwarm wurden täglich während der Flugzeit zu 5 bestimmten Uhrzeiten Proben heimkehrender Bienen genommen. Dabei wurde das Flugloch vorübergehend geschlossen. Wenn sich genügend Bienen (mehr als 20). am Flugloch gesammelt hatten, wurden sie eingefangen und in flüssigem Stickstoff abgetötet. Flüssiger Stickstoff wurde deswegen zur Tötung der Bienen herangezogen, weil durch das schnelle Töten und Einfrieren die Bienen nicht mehr in der Lage waren, den Honigblaseninhalt zu erbrechen. Diese Methode erschien uns günstiger als die in der Literatur angegebenen (Soehngen und Jay, 1974; Demianovizc, 1978). Nach dem Abtöten wurden die Bienen bei $-18^{\circ} \mathrm{C}$ in einer Gefriertruhe bis zur Bearbeitung der Proben aufbewahrt.

Vom Versuchsvolk wurden an Tagen mit Bienenflug solange Bienenproben genommen, bis verdeckelte Honigvorräte auf den inzwischen ausgebauten Mittelwänden eingelagert waren. $\mathrm{Zu}$ Versuchsende wurden vom Volk Honigproben durch Herauspipettieren aus der Wabe entnommen und bis zur Untersuchung bei $-18^{\circ} \mathrm{C}$ gelagert.

\section{Aufarbeitung der Proben}

Für diese Untersuchung wurden drei Kategorien von Daten herangezogen.
1. Von den Bienen wurde die Honigblase herauspräpariert und anschließend das Zuckerspektrum bestimmt.

Die Zuckeranalyse wurde mittels HPLC durchgeführt (Thean und Funderburk, 1971; Severson et al, 1983).

Die Honigblaseninhalte wurden in einem Eppendorfhütchen in $0,1 \mathrm{ml}$ Wasser gelöst bzw von Honig eine 1\%ige Lösung hergestellt, abzentrifugiert (Biofuge) und $20 \mathrm{mcl}$ der überstehenden Lösung in das HPLC-System injiziert. Als Trennsystem wurde eine Amino-HPLCSäule, Sperisorb S5 NH2 $250 \times 4$, mit Vorsäule, Sperisorb $\$ 1040 \times 4$, verwendet. Laufmittel : entgastes und filtriertes Acetonitril-WasserPutrescin-Gemisch $\quad(70: 30: 0,02)$; Elutionsgeschwindigkeit : $1 \mathrm{ml} / \mathrm{min}$; Detektion : Differenzialrefraktometer; Auswertung : Integrator.

Zur Überprüfung der Integrationsparameter wurden verschiedene Konzentrationen von Zukkerstandards injiziert und ausgewertet. Die Abweichungen lagen im Fehlerbereich der $\mathrm{Me}$ thode.

Bei der Auswertung wurden die Zuckeranteile von Fructose, Glucose, Saccharose, Melezitose einzeln und die anderen Zucker gesamt quantifiziert. Die Zuckermenge wurde hier nicht festgehalten. Probenumfang : 52 Bienen, verteilt (gezielt ausgesucht) auf die 9 wichtigsten Trachtarten (Tabelle I).

2. Wenn die Anzahl der Bienen in der Probe ausreichte bzw in der Probe eine ausreichende Anzahl erfolgreicher Sammlerinnen gefunden werden konnte, wurden pro Probe von den Honigblaseninhalten von mindestens 10 Bienen folgende Daten erhoben : Gewicht in $\mathrm{mg}$, botanische Herkunft (anhand der Pollenanalyse des Honigblaseninhaltes vor der Zuckerbestimmung), Gesamtzucker in $\mathrm{mg}$ (photometrisch mit der Anthron-Methode nach Humer (1981) und Burgstaller (1985). War die botanische Herkunft eines Honigblaseninhaltes unklar (kein Pollen, keine Pilzsporen vorhanden oder Pollen mehrerer nektarblütiger Pflanzen in einem Honigblaseninhalt), so wurden diese Honigblaseninhalte als "nicht zuzuordnen" eingestuft. Stichprobenumfang : 452 zufällig ausgewählte Bienen. Die Bienenproben aus den Datenkategorien 1 und 2 stammten aus den gleichen ursprünglichen Stichproben.

3. Honigproben von drei verschiedenen Waben dieses Volkes wurden nach 1. auf ihr 
Zuckerspektrum untersucht. Als zusätzlicher Vergleich wurde auch das Zuckerspektrum einer reinen Melezitose-Honigprobe analysiert.

Die Daten (Prozentwerte der Zuckeranteile) wurden varianzanalytisch bzw mit dem Bonferroni-Holm-Test auf ihre Verschiedenheit in den Zuckerspektren zwischen den Trachtarten geprüft.

\section{ERGEBNISSE}

Die Ergebnisse sind in den Tabellen I und II und in der Abbildung 1 dargestellt.

Die statistische Auswertung der Daten ergab signifikante Unterschiede im Zuckerspektrum zwischen den Trachtarten zumin-

Tabelle I. Die Codierung der einzelnen Trachtarten. Die Trachtart 3 wurde nicht in die Berechnungen einbezogen. $1-9=$ Honigblaseninhaite verschiedener Trachtherkunft; 10 und $11=$ Honige. Das durchschnittliche Zuckerspektrum (in \%, \pm sd) der verschiedenen Trachtarten. FR : Fructose, GL : Glucose, $\mathrm{SA}=$ Saccharose, $\mathrm{ME}:$ Melizitose, $\mathrm{AN}=$ andere Zucker .

\begin{tabular}{|c|c|c|c|c|c|c|c|c|c|}
\hline & Trachtart & $n$ & $F R$ & $G L$ & $S A$ & ME & $A N$ & $F R: G L$ & $S A: M E$ \\
\hline & $\begin{array}{l}1 \text { Honigtau flüssig (die Honigblaseninhalte } \\
\text { waren noch nach einem Jahr flüssig) }\end{array}$ & 6 & $\begin{array}{l}14,25 \\
(2,43)\end{array}$ & $\begin{array}{r}6,94 \\
(0,73)\end{array}$ & $\begin{array}{l}49,42 \\
(5,56)\end{array}$ & $\begin{array}{l}19,99 \\
(4,10)\end{array}$ & $\begin{array}{c}9,41 \\
(2,22)\end{array}$ & $\begin{array}{c}2,16 \\
(0,41)\end{array}$ & $\begin{array}{c}3,07 \\
(0,74)\end{array}$ \\
\hline 2 & $\begin{array}{l}2 \text { Honigtau kristallisiert (die Honig- } \\
\text { blaseninhalte waren schon nach einer } \\
\text { Woche Tiefkühllagerung kristallisiert) }\end{array}$ & 9 & $\begin{array}{l}24,38 \\
(2,88)\end{array}$ & $\begin{array}{l}10,27 \\
(2,30)\end{array}$ & $\begin{array}{l}20,47 \\
(4,78)\end{array}$ & $\begin{array}{l}41,12 \\
(2,19)\end{array}$ & $\begin{array}{c}3,76 \\
(1,27)\end{array}$ & $\begin{array}{c}2,86 \\
(0,33)\end{array}$ & $\begin{array}{c}0,49 \\
(0,12)\end{array}$ \\
\hline 3 & $\begin{array}{l}3 \text { Wassersammlerin (nur eine Probe) } \\
\text { Zuckergehalt } 2,8 \%\end{array}$ & 1 & 37,66 & 20,08 & 42,26 & 0,00 & 0,00 & 1,88 & \\
\hline 4 & 4 Rubus idaeus L & 14 & $\begin{array}{l}55,87 \\
(1,08)\end{array}$ & $\begin{array}{l}39,58 \\
(1,08)\end{array}$ & $\begin{array}{c}1,74 \\
(0,76)\end{array}$ & 0,00 & $\begin{array}{c}2,89 \\
(1,24)\end{array}$ & $\begin{array}{c}1,42 \\
(0,04)\end{array}$ & \\
\hline 5 & 5 Cirsium-Form ( $C$ oleraceum $L$ ) & 9 & $\begin{array}{l}33,06 \\
(5,41)\end{array}$ & $\begin{array}{l}17,34 \\
(2,87)\end{array}$ & $\begin{array}{l}37,72 \\
(7,17)\end{array}$ & $\begin{array}{c}6,59 \\
(2,71)\end{array}$ & $\begin{array}{c}5,29 \\
(1,15)\end{array}$ & $\begin{array}{c}2,20 \\
(0,34)\end{array}$ & \\
\hline 6 & 6 Comp S-From (kleine Cirsium-Form) & 3 & $\begin{array}{l}52,85 \\
(6,12)\end{array}$ & $\begin{array}{l}32,40 \\
(2,48)\end{array}$ & $\begin{array}{c}8,50 \\
(5,08)\end{array}$ & 0,00 & $\begin{array}{c}6,25 \\
(3,19)\end{array}$ & $\begin{array}{c}1,63 \\
(0,12)\end{array}$ & \\
\hline & 7 Senecio fuchsii C GMEL & 3 & $\begin{array}{l}15,88 \\
(3,93)\end{array}$ & $\begin{array}{l}5,67 \\
(0,37)\end{array}$ & $\begin{array}{l}41,18 \\
(8,68)\end{array}$ & $\begin{array}{l}25,47 \\
(4,70)\end{array}$ & $\begin{array}{l}11,80 \\
(2,29)\end{array}$ & $\begin{array}{c}2,74 \\
(0,55)\end{array}$ & $\begin{array}{c}1,88 \\
(0,78)\end{array}$ \\
\hline 8 & 8 Atropa belladonna L & 5 & $\begin{array}{l}39,05 \\
(5,09)\end{array}$ & $\begin{array}{l}21,89 \\
(4,36)\end{array}$ & $\begin{array}{l}27,47 \\
(9,48)\end{array}$ & $\begin{array}{c}2,57 \\
(1,82)\end{array}$ & $\begin{array}{c}9,04 \\
(2,97)\end{array}$ & $\begin{array}{c}1,93 \\
(0,28)\end{array}$ & \\
\hline 9 & $\begin{array}{l}9 \text { Umbelliferae H-Form } \\
\text { (Heracleum sphondylium L ?) }\end{array}$ & 4 & $\begin{array}{l}47,83 \\
(5,52)\end{array}$ & $\begin{array}{l}33,01 \\
(4,67)\end{array}$ & $\begin{array}{l}14,84 \\
(8,47)\end{array}$ & 0,00 & $\begin{array}{c}4,32 \\
(2,23)\end{array}$ & $\begin{array}{c}1,49 \\
(0,12)\end{array}$ & \\
\hline 10 & Honige vom Versuchsvolk & 3 & $\begin{array}{l}43,92 \\
(0,56)\end{array}$ & $\begin{array}{l}30,98 \\
(0,69)\end{array}$ & $\begin{array}{l}2,77 \\
(0,59)\end{array}$ & $\begin{array}{l}15,86 \\
(1,07)\end{array}$ & $\begin{array}{c}6,46 \\
(0,89)\end{array}$ & $\begin{array}{c}1,42 \\
(0,01)\end{array}$ & $\begin{array}{l}0,18 \\
(0,05)\end{array}$ \\
\hline 11 & $\begin{array}{l}1 \text { Melezitose - Honig aus derselben } \\
\text { Trachtzeit von einem anderen } \\
\text { Trachistandort (eine Probe als Vergleich }\end{array}$ & 1 & $\begin{array}{l}33,21 \\
(0,00)\end{array}$ & 19,46 & 4,07 & 36,73 & 6,52 & 1,71 & 0,11 \\
\hline
\end{tabular}


Tabelle II. Zuckeranteil der Trachtarten mit untersuchtem Zuckerpektrum am Gesamteintrag für den Honig. " Cirsium oleraceum L Pollen wurden in der Routinearbeit nicht von der kleineren Comp SForm unterschieden. ${ }^{\star *} 22$ verschiedene Pflanzengruppen und (bei 12 Bienen) unbekannte Pollen. *** Keine Anhaltspunkte zu finden ob Honigtau oder Nektar.

\begin{tabular}{|c|c|c|c|c|}
\hline Trachtart & n Bienen & $\begin{array}{c}\text { Zucker \% } \\
\text { Honigblasen- } \\
\text { inhalt }\end{array}$ & $\begin{array}{l}\text { Zucker mg pro } \\
\text { Biene }( \pm s d)\end{array}$ & $\begin{array}{l}\text { \%-Anteil am } \\
\text { gesamten } \\
\text { Zuckerertrag }\end{array}$ \\
\hline 1 & 96 & $\begin{array}{c}65,29 \\
(11,90)\end{array}$ & $\begin{array}{c}27,52 \\
(10,75)\end{array}$ & 35,76 \\
\hline 2 & 65 & $\begin{array}{c}66,25 \\
(12,47)\end{array}$ & $\begin{array}{c}23,84 \\
(11,55)\end{array}$ & 20,98 \\
\hline 4 & 27 & $\begin{array}{c}24,89 \\
(15,13)\end{array}$ & $\begin{array}{l}06,16 \\
(6,45)\end{array}$ & 02,25 \\
\hline $5+6^{*}$ & 58 & $\begin{array}{c}44,10 \\
(19,50)\end{array}$ & $\begin{array}{l}10,98 \\
(9,27)\end{array}$ & 08,62 \\
\hline 7 & 25 & $\begin{array}{c}60,88 \\
(16,97)\end{array}$ & $\begin{array}{r}18,69 \\
(13,67)\end{array}$ & 06,33 \\
\hline 8 & 11 & $\begin{array}{c}38,45 \\
(21,20)\end{array}$ & $\begin{array}{c}10,06 \\
(10,24)\end{array}$ & 01,50 \\
\hline 9 & 14 & $\begin{array}{c}52,64 \\
(16,61)\end{array}$ & $\begin{array}{l}10,66 \\
(9,61)\end{array}$ & 02,02 \\
\hline \multicolumn{5}{|l|}{ Andere } \\
\hline \multicolumn{4}{|l|}{ Nicht } & 12,40 \\
\hline zuzuordnen ${ }^{\star * *}$ & 59 & & $\begin{array}{c}12,61 \\
(10,38)\end{array}$ & 10,07 \\
\hline \multicolumn{5}{|l|}{ Summe } \\
\hline $1-9$ & 296 & & 19,33 & 77,46 \\
\hline
\end{tabular}

dest bei einer Zuckerart zwischen allen Trachtarten $(P<0,05$; mit Ausnahme Trachtarten 4:9, 6:8 bzw 9 und 8:5, bzw 7 und 9). Von den 28 möglichen Vergleichen zwischen den verschiedenen Trachtarten unterscheiden sich nur 6 nicht signifikant.

Aus der Abbildung 1 sind die Unterschiede zwischen den einzelnen Trachtarten in bezug auf die jeweiligen Zuckerarten dargestellt.

Die Haupttrachtarten Honigtau flüssig und Honigtau kristallisiert (Tabelle I) sind voneinander und zu allen anderen Trachtpflanzen verschieden.

\section{DISKUSSION}

Die gesamte Probennahme erfolgte während einer sehr ergiebigen Honigtautracht, die durch keine trachtlosen Tage unterbrochen wurde. Dadurch war ein kontinuierlicher Zuckereintrag ohne höheren zwischenzeitlichen Zuckerverzehr des Volkes gegeben. Es kann daher angenommen werden, daß die bei den Untersuchungen erfaßte Zuckermenge von 7,39 g eine repräsentative Stichprobe der vom Versuchsvolk während des Versuchszeitraumes vorwiegend als Honigkränze über der Brut eingetragenen Honig (geschätzt 8 $\mathrm{kg}$ ) darstellt. 
Die Auswertung der Zuckerspektren erfolgte in der Art, daß Fructose, Glucose, Saccharose, Melezitose einzeln und die anderen Zucker gesamt quantifiziert wurden, da laut Gölz (1981 und 1982) die vier genannten Zuckerarten zB beim Honigtau mehr als $90 \%$ des Gesamtzuckers ausmachen. Auch in Waldhonigen machen diese 4 Zuckerarten im Durchschnitt $88 \%$ am Gesamtzuckeranteil aus (Bogdanov und Baumann, 1988). Nach Bogdanov und Baumann (1988) ergibt sich in der schwierigen Trennungsmöglichkeit von Melezitose und Erlose bei der von uns eingesetzten Methode eine Fehlerquelle. Die Autoren geben einen durchschnittlichen ErloseGehalt bei 38 Honigtauhonigen aus der Schweiz von ca $2 \%$ an. Die erwähnte Fehlerquelle dürfte daher bei unseren Untersuchungen eher gering sein.

Die Ergebnisse zeigen, daß die Zuckerspektren der verschiedenen Trachtarten zumindest bei einer Zuckerart signifikant verschieden sind. Die Unterschiede zwischen den Zuckerspektren der Honigblasen mit "Honigtau flüssig" und "Honigtau kristallisiert" sind auffallend groß und einheitlich. Das Verhältnis SA:ME-Gehalt ist hier am auffallendsten. Honigtau flüssig

Tabelle III. Gesamtanteil in \% der einzelnen Trachtarten an den untersuchten Zuckerarten im Vergleich zum Honig. " Siehe Tabelle II. "* Das Zuckerspektrum der Trachtarten, bei denen keine Messung des Spektrums vorlag bzw wenn der Honigblaseninhalt keiner Tracht (weder Honigtau noch Nektar, siehe auch Tabelle III) zuordenbar war, wurde entsprechend dem Durchschnitt der untersuchten Trachtarten aufgeteilt. *** Bei der Invertierung von SA bzw ME wurden die Restanteile entsprechend dem Anteil im Honig als nicht invertiert angenommen.

\begin{tabular}{|c|c|c|c|c|c|c|}
\hline \multirow{2}{*}{$\begin{array}{l}\text { Trachtart } \\
\text { (Code) }\end{array}$} & \multicolumn{5}{|c|}{ Zuckerart } & \multirow{2}{*}{$\begin{array}{c}\text { Gesamt- } \\
\text { anteil }\end{array}$} \\
\hline & $F R$ & $G L$ & $S A$ & $M E$ & $A N$ & \\
\hline 1 & 5,10 & 2,48 & 17,67 & 7,15 & 3,37 & 35,77 \\
\hline 2 & 5,11 & 2,15 & 4,29 & 8,63 & 0,79 & 20,97 \\
\hline 4 & 1,26 & 0,89 & 0,04 & 0,00 & 0,07 & 2,26 \\
\hline $5+6^{*}$ & 3,28 & 1,82 & 2,62 & 0,43 & 0,48 & 8,63 \\
\hline 7 & 1,00 & 0,36 & 2,60 & 1,61 & 0,75 & 6,32 \\
\hline 8 & 0,59 & 0,33 & 0,41 & 0,04 & 0,14 & 1,51 \\
\hline 9 & 0,97 & 0,67 & 0,30 & 0,00 & 0,09 & 2,03 \\
\hline \multicolumn{7}{|l|}{$\begin{array}{l}\text { Summe } \\
\text { untersuchter }\end{array}$} \\
\hline $\begin{array}{l}\text { Nicht } \\
\text { untersuchte }\end{array}$ & ו & 0,70 & 21,93 & 17,00 & 5,09 & $\pi, 4 \mathrm{y}$ \\
\hline Pflanzen ${ }^{* *}$ & 5,10 & 2,53 & 8,12 & 5,09 & 1,66 & 22,50 \\
\hline Summe gesamt & 22,41 & 11,23 & 36,05 & 22,95 & 7,35 & 99,99 \\
\hline $\begin{array}{l}\text { Summe nach } \\
\text { angenommener }\end{array}$ & & & & & & \\
\hline Invertierung ${ }^{* \star *}$ & 41,60 & 32,42 & 2,77 & 15,86 & 7,35 & 99,95 \\
\hline Honig (Durchschnitt) & 43,92 & 30,98 & 2,77 & 15,86 & 6,47 & 100,00 \\
\hline
\end{tabular}




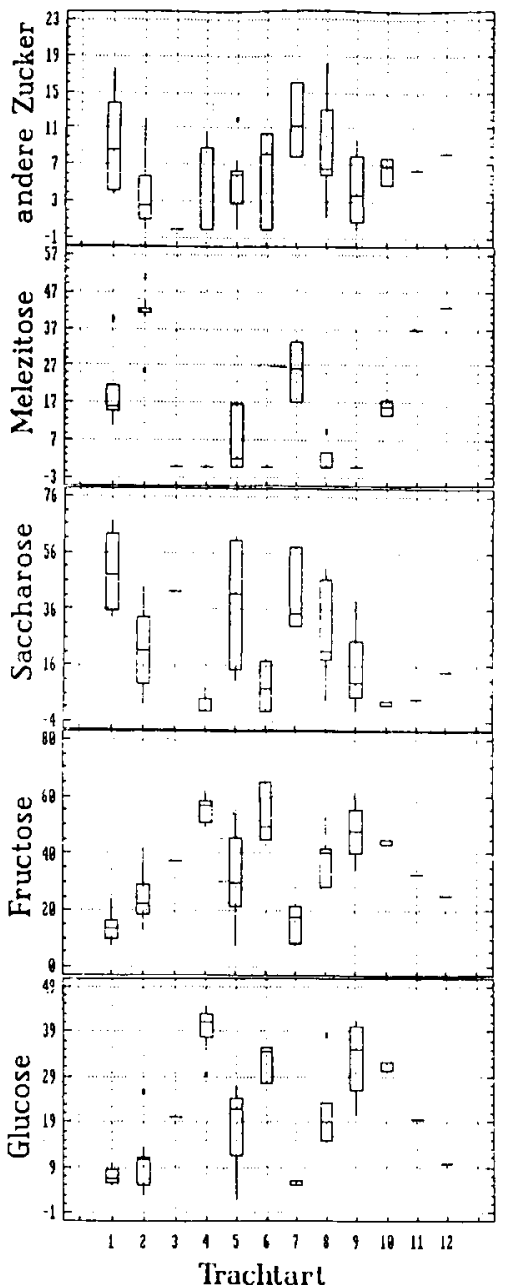

Abb 1. Die Zuckerspektren der einzelnen Trachtarten (Box- und Whisker-Plot). Die Skala für die einzelnen Zucker gibt den Prozentwert dieses Zuckers am Gesamtzucker an.

weist $49,42( \pm 5,56) \%$ SA : 19,99 ( \pm 4,10)\% ME und Honigtau kristallisiert $20,47( \pm 4,78)$ SA zu 41,12 ( $\pm 2,19) \% \mathrm{ME}$ auf. Es läßt sich errechnen, daß die Ursache der Kristallisation allein am absoluten Melezitosegehalt und nicht am Verhältnis SA:ME liegt. Diese Ergebnisse stimmen mit den bisherigen Angaben (Liebig, 1979; Gölz, 1981) überein.

Die Zuckerspektren der Honigblaseninhalte mit Nektar sind ebenfalls bei Berücksichtigung der botanischen Herkunft untereinander signifikant verschieden. Auffallend ist, daß auch im Nektar verschiedener Trachtpflanzen (besonders Senecio) ein hoher Anteil an Melezitose gefunden wurde $(25,47 \pm 4,70 \%)$. Hier muß allerdings auch der reine Nektar direkt von der Pflanze gesammelt und analysiert werden, wie dies bei verschiedenen anderen Pflanzen bereits geschehen ist (Severson et al, 1983; Pais et al, 1986).

Nach Soehngen and Jay (1974), Juntawong (1989) nehmen Bienen einen Proviant mit auf den Sammelflug. Die Menge beträgt sehr konstant um $5 \mathrm{mg}$ pro Biene. Bei dem hohen durchschnittlichen Gewicht des Einzeleintrages pro Biene (Honigblasengewicht $29,07 \pm 16,26 \mathrm{mg}$ und Zuckereintrag $16,35 \pm 13,10 \mathrm{mg}$ pro Biene) ist dadurch keine große "Vertälschung" des tatsächlichen Zuckerspektrums anzunehmen. Ungeklärt ist, ob dieser Proviant bei guter Tracht aus einem Rest des Sammelgutes vom vorhergehenden Ausflug oder aus den Vorräten des Volkes stammt. Aufgrund unserer Ergebnisse scheint ersteres der Fall zu sein, da weder das Zuckerspektrum (vor allem der Gehalt an ME, GL, SA) und auch die Eintragsleistung nektarsammelnder Bienen zB nicht mit dem Honigblaseninhalt honigtausammelnder Bienen übereinstimmt (Tabelle I und II). Auch der Pollen bei nektarsammelnden Bienen stammte in der Regel nur von einer Pflanze, so daß eine generelle Verproviantierung aus den Stockvorräten nicht anzunehmen ist.

Warum der Honigblaseninhalt zB von Bienen mit Senecio-Nektar trotz des zum Teil hohen Melezitosegehaltes nicht kristallisiert war, ist unklar. Die kristallisierten Proben waren alle aufgrund der Pilzsporen 
und den im Honigblaseninhalt gefundenen Pinus- bzw Picea-Pollen nur dem Honigtau zuzuordnen.

Es wurde das Zuckeraufkommen für den Gesamtzucker des Honigs anhand der 452 untersuchten Bienen (7388 mg Zucker wurden erfaßt) aufgrund des Zuckerspektrums der 52 analysierten Bienen hochgerechnet (Tabelle III). Daraus läßt sich ein annähernd korrektes Zuckerspektrum des Gesamteintrages ableiten. Dieses hochgerechnete Zuckerspektrum wurde nach einer zu erwartenden Invertierung vom Großteil der Saccharose und einem kleineren Teil der Melizitose in Tabelle III mit dem Durchschnitt des Zuckerspektrums der drei untersuchten Honigproben verglichen.

Es konnte bei dieser Hochrechnung das Zuckerspektrum von $77,46 \%$ des gesamten geschätzen Zuckereintrages erfaßt werden. Da die restlichen $22,5 \%$ des Zukkereintrages in ihrer botanischen Herkunft sehr breit gestreut waren oder überhaupt keiner Tracht (weder Honigtau noch Nektar) zugeteilt werden konnten (Tabelle II), kann man annehmen, daß das Zuckerspektrum dieser Honigblasenherkünfte annähernd dem Durchschnitt der untersuchten Tracht entspricht. Daß diese Annahme berechtigt ist, zeigt einerseits die Übereinstimmung in Honigblasengewicht und Zukkerprozent mit anderen Blütenpflanzen und die gute Übereinstimmung des geschätzten Zuckerspektrums mit dem Zukkerspektrum des Honigs.

Zum Vergleich der Zuckerspektren der Honigblaseninhalte (geschätzt) und der Honige kann folgendes bemerkt werden : Die gute Übereinstimmung läßt den Schluß zu, daß die Untersuchungen repräsentativ waren. Das Zuckerspektrum des Honigs ist daher geeignet, eine Aussage über die Trachtherkunft zu machen. Dies deckt sich auch mit den Aussagen von Maurizio (1964). Die signifikanten Unterschiede in den Zuckerspektren zwischen den einzelnen Trachtarten bestätigen auch die Annahme, daß man anhand des Pollenspektrums des Honigblaseninhaltes relativ einfach die Trachtherkunft und damit auch den wahren Trachtwert einer Pflanze sehr gut erkennen kann. Diese Frage wird in einer weiteren Arbeit genauer untersucht werden. Bei künftigen Arbeiten wäre es von Vorteil, mehr als nur die vier Zuckerarten zu analysieren. Auch der Einfluß der Transglucosidasen und die Bildung weiterer Zuckerarten wären zu berücksichtigen.

Die Ergebnisse zeigen auch, daß das Zukkerspektrum der drei untersuchten Honige (von drei verschiedenen Waben) sehr einheitlich ist. Daraus ist zu erkennen, daß die Bienen offenbar mehr als $90 \%$ der eingetragenen Saccharose invertieren können $(36,05 \%$ SA eingetragen, $2,77 \%$ "Rest" SA im Honig). Dies ist eine Bestätigung der Untersuchungen von Maurizio (1961) und Deifel (1985). Dagegen scheinen die Bienen mit der Invertierung der ME eindeutig Probleme zu haben. Der geschätzte Anteil der ME von $22,72 \%$ am Gesamtzuckereintrag konnte nur auf $15,86 \%$ im Honig invertiert werden. Das entspricht einer Spaltung von nur rund $30 \%$ der eingetragenen Melezitose unter den gegebenen Verhältnissen. Gontarski (1954), Maurizio (in Kloft et al, 1985) und White and Mahrer (1953) geben zwar für die Arbeitsbienen im Pharynxdrüsensekret Fermente an, die zur Spaltung der Melezitose dienen. Diese Fermente dürtten aber nicht ausreichen, um in einem großen Angebot an melezitosehaltigem Zuckereintrag einen Großteil der Melezitose zu spalten. Diese Annahme wird auch dadurch bestätigt, daß die ebenfalls untersuchte Honigprobe eines Melezitosehonigs noch $36,73 \%$ Melezitose im Zuckerspektrum aufwies (Tabelle I, Trachtart 11). Im Vergleich dazu weist der Honigtau kristallisiert ("Melezitose-Honigtau") im Durchschnitt $41,12 \%$ Melezitose auf. Die Honigprobe (“Trachtpflanze" 11) mit 36,73\% Melezi- 
tose war in der Wabe vollständig auskristallisiert. Wäre reiner Honigtau kristallisiert eingetragen worden, käme dies einer Aufspaltung der Melezitose von rund 10,7\% gleich. Das jeweilige Trachtspektrum könnte einen Einfluß auf den Grad der Aufspaltung der Melezitose haben. Die praktischen Erfahrungen bei Melezitosetrachten lassen dies ebenfalls vermuten.

\section{Summary - Investigation of sugar spectrum in honey-sac content and} honey. The sugar spectrum was investigated by HPLC in honey-sac contents of 52 fieldbees and in honey of a specially prepared colony. The origin of the honey-sac content was determined from the pollen species. The sugar spectrum has a typical frequency from the most frequent honey plants. A good correlation between the honey-sac content of the fieldbees and the honey produced in the experimental hive was found by consideration of enzyme effect (tables II and III). Liquid honeydew collected had a high sucrose content $(49.42 \pm 5.56 \%)$, but honeydew cristallized in the honey-sac of the stored bees ($18^{\circ} \mathrm{C}$ ) within less than 2 wk had a lower sucrose $(20.47 \pm 4.78 \%)$ but a much higher melecitose content $(41.12 \pm 2.19 \%$ to $19.99 \pm 4.10 \%$ in honeydew liquid). In this colony more than $90 \%$ of sucrose and only $30 \%$ of the melezitose collected from the bees could be inverted. The sugar spectrum of the honey-sac content provides information about the botanical origin. On the basis of the sugar spectrum of the honey the botanical origin can also be estimated.

sugar spectrum / honey / honey-sac content / botanic origin

Résumé - Etude comparative du spectre des sucres du jabot des abeilles et de celui du miel. On a déterminé individuellement par HPLC le spectre des sucres du jabot de 52 butineuses rentrant dans leur ruche et du miel d'une colonie expérimentale. L'origine botanique du contenu du jabot a été déterminée par analyse pollinique. Le spectre des sucres présente des différences significatives entre les plantes mellifères les plus importantes, pour au moins un type de sucre (tableau I, fig 1). On a trouvé une bonne correspondance entre le spectre des sucres de l'ensemble des contenus du jabot des butineuses et celui du miel produit par la ruche expérimentale (tableaux II et III). Le miellat liquide récolté possédait une forte teneur en saccharose $(49,42 \pm 5,56 \%)$ mais le miellat cristallisé dans le jabot des abeilles conservées à $-18^{\circ} \mathrm{C}$ pendant moins de 2 semaines avait une teneur en saccharose plus faible $(20,47 \pm 4,78 \%)$ et une teneur en mélézitose beaucoup plus élevée $(41,12 \pm 2,19$ contre $19,99 \pm 4,10 \%$ dans le miellat liquide) (tableau I).

Dans cette colonie, plus de $90 \%$ du saccharose et près de $30 \%$ seulement du mélézitose récolté par les abeilles a pu être inverti. Le spectre des sucres du contenu du jabot renseigne sur son origine botanique. L'origine botanique du miel peut également être évaluée d'après le spectre des sucres.

\section{miel / spectre des sucres / contenu du jabot / origine botanique}

\section{LITERATUR}

Bogdanov S, Baumann E (1988) Bestimmung von Honigzuckern mit HPLC. Mitt Geb Lebensm Hyg 79, 198-206

Burgstaller H (1985) Die bienenwirtschaftliche Bedeutung des Löwenzahnes. Diplomarbeit an der Universität für Bodenkultur, Wien

Demianowicz Z (1978) Inhalt der Honigblase der Arbeitsbienen von Apis mellifera $L$ während 
der Tannenhonigtautracht. Apidologie 9, 117122

Deifel A (1985) Zur Kenntnis der Saccharose im Honig. Dissertation an der Universität Stuttgart-Hohenheim

Gölz H (1981) Der Melezitosegehalt im Honigtau verschiedener Lachnidenarten. Diplomarbeit Universität Stuttgart-Hohenheim

Gölz H (1982) Der Melezitosegehalt im Honigtau verschiedener Lachnidenarten. Apidologie 13 (2), 89-90

Gontarski H (1954) Fermentbiologische Studien an Bienen. Das physochemische Verhalten der Kohlehydratspaltenden Fermente : a. Invertierende Enzyme. Verh Ges Angew Entomol 186-197

Handel E van, Haeger JS, Hansen CW (1972) The sugar of some Florida nectars. Am J Bot 59(10), 1030-1032

Humer J (1981) Nektar- und Pollenuntersuchung an sammelnden Honigbienen. Diplomarbeit, Universität für Bodenkultur, Wien

Juntawong $N$ (1989) Verweildauer von Pollen bestimmter botanischer Herkunft in der Honigblase und im Honig bei Apis mellifera carnica L. Dissertation, Universität für Bodenkultur, Wien

Kloft W, Maurizio A, Kaeser W (1985) Waldtracht und Waldhonig in der Imkerei. Ehrenwirth, München

Liebig G (1979) Gaschromatographische und enzymatische Untersuchungen des Zuckerspektrums des Honigtaues von Buchneria pectinatae (Nördl). Apidologie 10, 213-225
Low NSH, Neison DL, Sporns P (1988) Carbohydrate analyses of Western Canadian honeys and their nectar sources to determine the origin of honey oligosaccharides. J Apic Res 27, 245-251

Maurizio A (1961) Fermentwirkung während der Überwinterung der Ligusticarasse. Insectes Soc 8, 125-175

Maurizio A (1964) Das Zuckerbild blütenreiner Sortenhonige. Ann Abeille 7, 289-299

Pais MSS, Chaves das Neues HJ, Vasconcelos MPA (1986) Amino acid and sugar content of the nectar exudate from Limodorum abortivum (Orchidaceae). Comparison with Epipactis atropurpurea nectar composition. Apidologie 17, 125-136

Severson DW, Ericson EH (1983) High performance liquid chromatography of carbohydrates in cucumber nectar. $J$ Apic Res 22 , 158-162

Sihag RC, Kapil RP (1984) Foraging strategies of honeybees as determined by quality and quantity of nektar. $5^{e}$ Symp Int Pollinisation, Versailles 1983, INRA, 51-59

Soehngen U, Jay C (1974) Studies on the honey-sac contents and pollen loads of honeybees. 2. Honey-sac contents of foraging bees. J Apic Res 13, 199-206

Thean JE, Funderburk WC (1971) High Pressure Liquid Chromatographic Determination of Sucrose in Honey. $J$ Assoc Off Agric Chem 60, 838-841

White JW, Mahrer J (1953) Transglucosidation by honey invertase. Arch Biochem Biophys $42,360-367$ 